\title{
Research on Air Traffic Center Control Effectiveness Evaluation Based on Cloud Model
}
Weijun PAN ${ }^{1, a}$, Yuexiao PAN ${ }^{1, b *}$, Chuyang YANG ${ }^{1, c}$, Qinghai ZUO ${ }^{1, d}$, Jie LUO $^{1, e}$, Qingyu ZHANG ${ }^{1, f}$

${ }^{1}$ Department of Air Traffic Management, Civil Aviation Flight University of China, Guanghan, 618300, China

bemail:963370391@qq.com, cemail: 2425917402@qq.com

\begin{abstract}
Keywords: Air Traffic Control Center; Index System; Cloud Theory; Effectiveness Evaluation; Forward Normal Cloud Generator;
\end{abstract}

\begin{abstract}
In order to convert the qualitative description to quantitative concept of effectiveness evaluation of ATCC (Air Traffic Control Center), this paper selects artificial, automation equipment, management and operation environment as effectiveness evaluation factors based on cloud theory, and established a performance evaluation index. Taking a typical ATCC as example, the comment set of each factor is converted into cloud model. The comprehensive cloud picture of factors is produced by forward normal cloud generator. The experiment shows that the method can overcome fuzziness and randomness in the process of traditional evaluation and can accurately reflect control center's effectiveness evaluation level.
\end{abstract}

\section{Introduction}

Air traffic control center is a composition center of multi-level and multivariate composite made by various factors [1]. At present, the related scholars has carried out some existing research of the efficiency, Bai Songhao first introduced the concept of efficiency to the air traffic control areas and applied the neural network method to rough evaluation of air traffic control system efficacy in 2004[2]. Then he tried to follow the example of Europe and the United States to establish effectiveness evaluation index system which is suitable for China's characteristics is shown in: including "security, delay, cost efficiency and effectiveness can be forecast ", but it belongs to the ideal evaluation index system, its refining indicators are not corresponding to statistics [3-4]; These methods only gave a comprehensive evaluation results, the efficiency evaluation cannot completely solved the uncertainty in the process of quantitative and qualitative concept conversion, it means the fuzziness and randomness in the process of transformation.

\section{Design of Normal Model --- Cloud Theory}

Definition $U$ is a certain domain of discourse, $C$ is a qualitative concept on $U$ 。 that is : $U \rightarrow[0,1], \forall x \in U_{x} x \rightarrow \mu(x)$ 。 So the distribution of $x$ on the theory of domain $U$ called cloud. , recorded $C\left(E x_{r} E n_{r} H e\right)$, fig. 1 。 $x$ is expectation, $E n$ is the qualitative concept of randomness ,He is entropy, So each $x$ is cloud droplets [5-6]。

$$
\begin{aligned}
& \text { If } \mathrm{X} \sim \mathrm{N}\left(H e^{\gamma}\right)^{2}, E n^{i} \sim \mathrm{N}\left(E_{n} H_{e}\right)^{2} \text {, then } \\
& u=e^{-\left[\frac{-\left(x-E_{x}\right)^{2}}{2\left(E_{n}\right)^{2}}\right]}
\end{aligned}
$$

The distribution of $x$ on the theory of domain $U$ called normal cloud. 


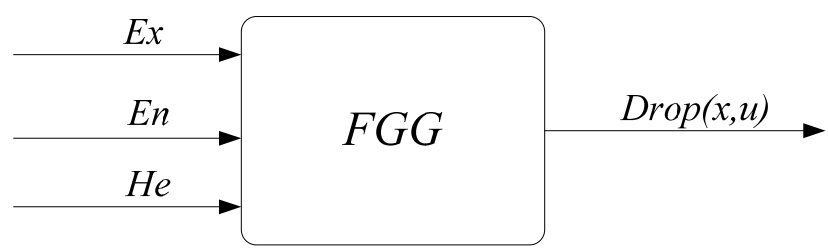

Fig.1 forward normal cloud generator

\section{(1) Algorithm of forward normal cloud generator $\operatorname{FCG}\left(E_{x^{\prime}} E_{n}, \boldsymbol{H}_{e}\right)$ :}

(1) normal random number $E e^{\prime}$ is generated by expectations $E$ 'r and variance $H \mathbf{s}^{2}$;

(2) normal random number $x$ is generated by expectations $E x$ and variance $H n^{2}$;

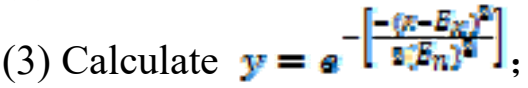

(4) Make (x, y) become a cloud droplets in the domain of discourse;

(5) Repeat steps (1) (4) until obtain the required cloud droplets in the domain of discourse.

Backward cloud generator can realize conversion between quantitative value and qualitative language value, that is, find out eigenvalue of three forward cloud generator from the given cloud sample, so as to realize the qualitative evaluation of the sample data.

(2) Algorithm of backward normal cloud generator $\mathrm{FCG}^{-1}\left(\boldsymbol{E}_{x}, \boldsymbol{E}_{n}, \boldsymbol{H}_{\mathrm{e}}\right)$ :

(1) According $x_{i}$, calculate the sample mean of this figures $\mathrm{X}=\frac{1}{n} \sum_{i-1}^{n} x_{1}$, 1-rank sample absolute center distance $\frac{1}{n} \sum_{i-1}^{n}\left|x_{1}-\bar{X}\right|$, sample variance is $\mathrm{g}^{2}=\frac{1}{\mathrm{n}-1} \mathrm{Z}_{i-1}^{n}\left(\mathrm{x}_{1}-\bar{X}\right)^{2}$;

(2) $E_{x}=\bar{X}$;

(3) $E_{n}=\sqrt{\frac{\pi}{2}} \times \frac{1}{n} \sum_{i=1}^{n}\left|\mathrm{~s}_{j}-E_{x}\right|$;

(4) $H_{\mathrm{v}}=\sqrt{{\delta^{2}-E_{\mathrm{n}}{ }^{2}}^{2}}$

Therefore, when the samples of cloud droplets is $x_{i}$, it can get description of cloud which is $\left(E_{x,} E_{n,} H_{\varepsilon}\right)$.

\section{Effectiveness evaluation method based on cloud model}

According to index set of the established index system, each index judgment matrix of each index set is given to calculate the index of the relative weights of the index set in its place and converted into a weight cloud. Using expert scoring method is a layer of the evaluation of indicators, and converted to evaluate cloud, a cloud of index layer, the weight of each index and evaluation weight fitting operation of cloud, it is concluded that the index evaluation cloud of rule layer, and so on, finally concluded that control center system's overall effectiveness [7]. 


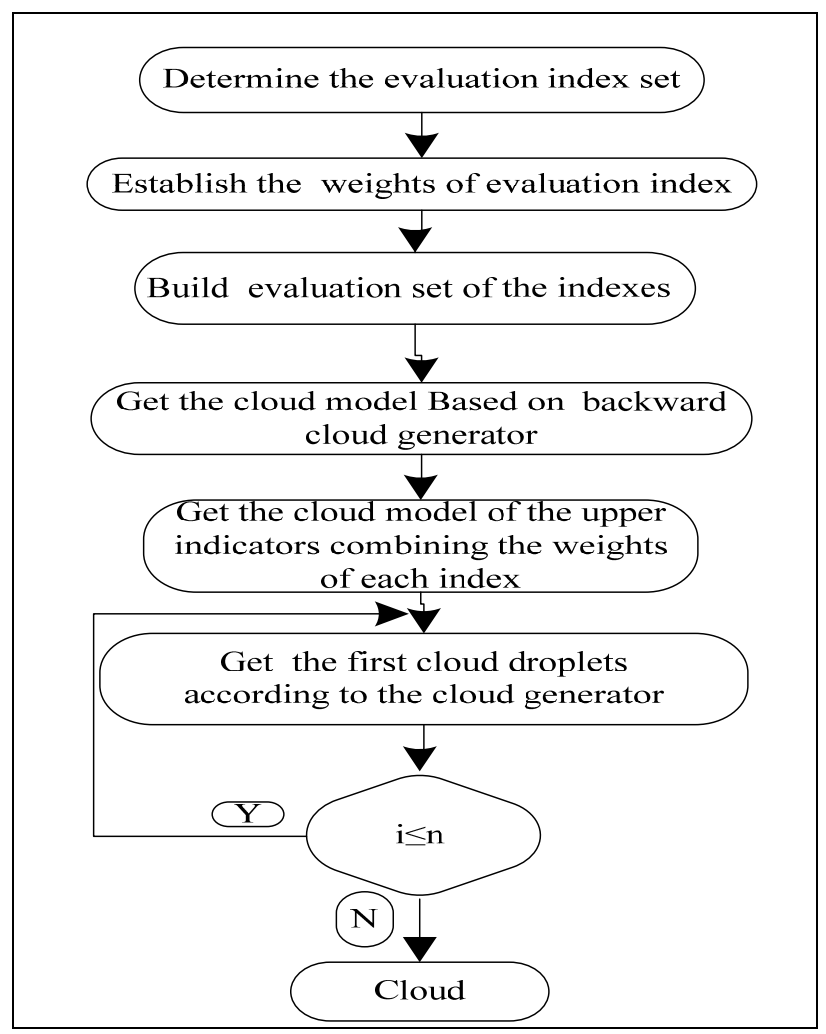

Fig.2 Assessment Procedure

\section{Build Cloud Evaluation System}

Establish air traffic control center effectiveness evaluation index: attribute set of first evaluation factor: remember $U=\{U 1, U 2, U 3, U 4\}=$ artificial factors, automation equipment, management factors, operation environment $\}$. Each evaluation factor has several secondary evaluation factors, as is shown in figure 2.

\section{Test results}

Using analytic hierarchy process (AHP) calculates the weight of each factor for air traffic control center effectiveness evaluation system. Weights of index factor for air traffic control center effectiveness evaluation are shown in table 1

Table 1 Weight of index factor for air traffic control center effectiveness evaluation

\begin{tabular}{|c|c|c|c|}
\hline First Level Evaluation Index U & Weights & Second Level Evaluation Index & Weights \\
\hline \multirow{2}{*}{ artificial } & \multirow{2}{*}{0.3} & Work quality & 0.1 \\
\hline & & Work quality & 0.1 \\
\hline \multirow{4}{*}{ automation equipment } & \multirow{4}{*}{0.3} & Surveillance capability & 0.1 \\
\hline & & Command lead & 0.1 \\
\hline & & Notification coordination & 0.1 \\
\hline & & Identification analysis & 0.1 \\
\hline \multirow{2}{*}{ Management } & \multirow{2}{*}{0.2} & Rules and regulations & 0.1 \\
\hline & & Safety awareness & 0.1 \\
\hline \multirow{2}{*}{ operation environment } & \multirow{2}{*}{0.2} & Airspace sector structure & 0.1 \\
\hline & & Flow & 0.1 \\
\hline
\end{tabular}


Table 2 Cloud model parameters of second level evaluation index

\begin{tabular}{c|c}
\hline Second Level Evaluation Index & $\left(\boldsymbol{F}_{\boldsymbol{z}}, \boldsymbol{F}_{\boldsymbol{n}}, \boldsymbol{H}_{\boldsymbol{\varepsilon}}\right)$ \\
\hline Work quality & $(9.567,0.900,0.557)$ \\
\hline Work quality & $(9.514,0.983,0.500)$ \\
\hline Surveillance capability & $(9.465,1.065,0.468)$ \\
\hline Command lead & $(9.422,1.127,0.415)$ \\
\hline Notification coordination & $(9.493,1.021,0.491)$ \\
\hline Identification analysis & $(9.449,1.095,0.502)$ \\
\hline Rules and regulations & $(9.410,1.148,0.444)$ \\
\hline Safety awareness & $(9.415,1.142,0.439)$ \\
\hline Airspace sector structure & $(9.379,1.197,0.439)$ \\
\hline Flow & $(9.413,1.152,0.497)$ \\
\hline
\end{tabular}

After get the digital eigenvalue of cloud model of bottom evaluating index, we can obtain the evaluation results of upper indicators combining weight value of bottom index. The formula is as follows:

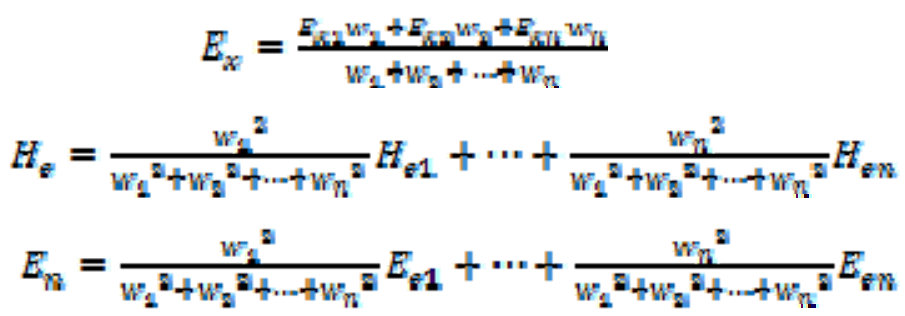

Using the above formula $(2 \sim 4)$, combined to the cloud of four sub-indexes respectively, we can get all the digital eigenvalue of cloud model of first indicators, as shown in table 3 。

Table 3 cloud model parameters of first level evaluation index

\begin{tabular}{c|c}
\hline First Level Evaluation Index & $\left(E_{x}, E_{n}, H_{w}\right)$ \\
\hline artificial & $(9.540,0.941,0.528)$ \\
\hline automation equipment & $(9.443,1.096,0.442)$ \\
\hline Management & $(9.451,1.088,0.479)$ \\
\hline operation environment & $(9.402,1.164,0.458)$ \\
\hline
\end{tabular}

$E x=9.453, E n=1.093, H e=0.474$ 。

Using digital eigenvalue and forward normal cloud generator algorithm, we can get the evaluation cloud, as is shown in figure 3

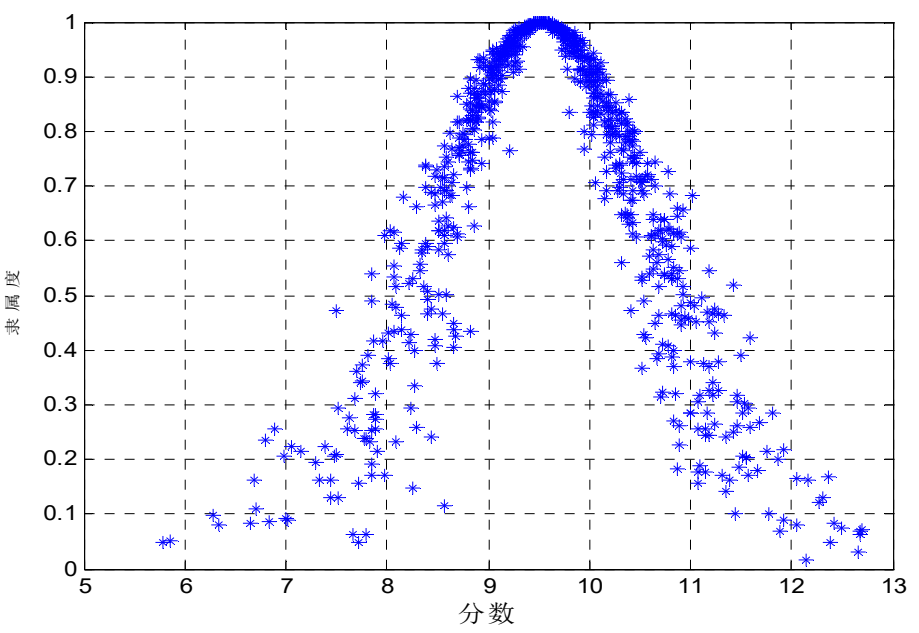

Fig.3 the cloud of Air Control Center Effectiveness evaluation

From figure 3, we can see cloud model expectations $E x$ of the efficiency evaluation results is 
9.453, which fall in the interval $(9,10)$, and cloud droplets distributed most densely populated in the range, therefore it can be concluded that the results of air traffic control center effectiveness evaluation "satisfaction".

\section{Conclusion}

Air traffic control center effectiveness evaluation method based on cloud theory is present the evaluation results of various indicators by cloud model, and combine the fuzziness and randomness together organically, and realize the natural transformation between qualitative linguistic value and quantitative numerical, thus compared with the traditional evaluation method, and can reflects the service level of the effectiveness evaluation more accurately.

\section{Acknowledgement}

This research was sponsored by the Graduate Innovation Foundation of Civil Aviation Flight University of China (Project No.X2015-21) and Nature Science Foundation of Henan Province (Project No. 71573184).

\section{Reference}

[1] Cheng Lei, Duo-he Ma, Hong-qi Zhang, Li-ming Wang, Jean J. Loiseau. Moving Target Network Defense Effectiveness Evaluation Based on Change-Point Detection [J]. Mathematical Problems in Engineering, 2016, 2016:.

[2] Bai song hao. Calculation model and method of System performance index and effectiveness factors [J]. System Engineering Theory and Practice, 2010, 30(11):2112-2220.

[3] Bai song hao. Air Control center system effectiveness evaluation based on neural network [J]. Telecommunication Engineering, 2004, 6:125-128

[4] Bai yonglei. Analysis and design ATC automation system [D]. Journal of Sichuan University, 2005.

[5] Pilar Pineda-Herrero,Esther Belvis,Victoria Moreno,Maria M. Duran-Bellonch,Xavier Úcar. Evaluation of training effectiveness in the Spanish health sector [J]. Journal of Workplace Learning, 2011, 235:

[6] Susan A. Moore, Michelle Walker. Progressing the Evaluation of Management Effectiveness for Protected Areas: Two Australian Case Studies [J]. Journal of Environmental Policy \&amp; Planning,2008,104:

[7] Qi Liu,Jun Cui, HongDong Zhao, Hong Shen. A Soft Fault-concept Diagnosis Method of Analog Circuits Based on Cloud Model Theory [J]. Journal of Networks, 2013, 87. 